\title{
Chlamydial infection in female lower genital tract and its correlation with cervical smear abnormalities
}

\author{
Peitsidis $\mathrm{P}^{1,2}$, Kalmantis $\mathrm{K}^{2}$, Peitsidou $\mathrm{A}^{2}$, Zervoudis $\mathrm{S}^{2}$, Papaspyrou $\mathrm{I}^{2}$, Georgoulias $\mathrm{N}^{2}$, Kioses $\mathrm{E}^{2}$
}

Department of Obstetrics and Gynaecology, Alexandra Hospital, Athens, Greece. panagiotis_pp@yahoo.com

\begin{abstract}
Background: It is widely known that infection by Chlamydia trachomatis (CT) is the most common curable sexually transmitted infection (STI) among the young population.

Chlamydial cervicitis has been suspected in the past to be a risk factor for squamous intraepithelial neoplasia (SIL) and cervical cancer on the basis of case-control comparisons of serological tests.

Methods: A total of 110 women with symptomatic cervicitis were examined and samples for microbiologic detection of Chlamydia trachomatis antigen were obtained. Colposcopic, cytologic and microbiologic analyses were performed. All results were statistically analysed and correlated.

Results: 41 patients (37.2\%) were found positive for Chlamydia trachomatis antigen (Group I) and 69 patients $(62.8 \%)$ were negative for Chlamydia trachomatis antigen (Group II). When compared to group II, the chlamydia-infected group I of women presented a larger percentage of dyspareunia (60\%), and the dysuric complaints were more common. The chlamydia-positive group revealed a higher incidence of atypical squamous metaplasia (52.5\% vs $27.9 \%$ ). Among the women with positive ELISA for chlamydia, a higher percentage of low-grade SIL was observed (6.3\% vs $3.6 \%)$.

Conclusions: Chlamydial infection, especially chlamydial cervicitis occurs often among young women of reproductive age. In these women, a good clinical assessment is necessitated including previous clinical history record, as well as detailed microbiologic, cytologic and colposcopic evaluations (Tab. 5, Ref. 19). Full Text in PDF www.elis.sk.

Key words: infection by Chlamydia trachomatis, sexually transmitted infection, cervicitis.
\end{abstract}

It is widely known that the infection by Chlamydia trachomatis (CT) is the most common curable sexually transmitted infection (STI) among the young population (1). Several studies have evaluated the outcomes of Chlamydia trachomatis infection in the National Health Service (2, 3). Chlamydial cervicitis has been suspected to be a risk factor for SIL and cervical cancer on the basis of case-control comparisons of serological tests (4). It has been shown that $\mathrm{CT}$ infection is more frequent in females with CIN than in females with normal cytological findings (5). However, Takac et al (6) indicate that CT infection is not related to the development or even to promotion of CIN. CT infections can cause several complications such as pelvic inflammatory disease (PID) and infertility $(7,2)$. Chlamydia trachomatis is a Gram-negative obligate intracellular bacterium with an incubation period of seven to 21 days, and a growth cycle of approximately $48 \mathrm{~h}$. It has a limited host range, infecting only human epithelial cells (8). To

${ }^{1}$ Department of Obstetrics and Gynaecology, Royal Free Hospital, London, United Kingdom, ${ }^{2}$ Department of Obstetrics and Gynaecology, Alexandra Hospital, Athens, Greece, and ${ }^{3}$ Department of Pathology, Alexandra Hospital, Athens, Greece

Address for correspondence: Dr. P. Peitsidis, Proussis 22 Street, Nea Smyrni, Athens, Greece. Post code: 17123 Phone: +302109334293 , Fax: +302109359391

Acknowledgement: We would like to thank the nursing and laboratory personnel of our hospital for their assistance in completing the current study. date, 18 different serotypes have been identified, 11 of which are predominantly isolated from the urogenital tract (serotypes $\mathrm{D}, \mathrm{Da}$, E, F, G, Ga, H, I, Ia, J, and K) (9). This prospective study assesses the local prevalence of Chlamydia trachomatis at our colposcopy clinic, and its correlation with cervical smear abnormalities.

\section{Material and methods}

A total of 110 non-consecutive women with symptomatic cervicitis participated in the current study. Participants visited the outpatient Gynaecology department of Alexandra General Hospital in Athens in period of December 2001 - June 2003, primarily complaining of pelvic pain and/or vaginal discharge. All women who received antibiotic treatment within three weeks prior to their visit were excluded from our study. Pregnant women were also excluded from the study. After informed consent had been obtained, a questionnaire was administered which included questions concerning the age at first sexual intercourse, gynaecological and obstetric history, duration and regularity of menstrual cycle, number of sexual partners, current use of oral contraceptives, and previous colposcopic evaluations. All these women underwent a collection of culture samples for Chlamydia trachomatis and STDs (Gonorrhea and Mycoplasma) from the endocervical canal. Following the previous procedure, the next step was to collect the cervical smears and to evaluate the colposcopic examination. Finally, all women under- 
went a general physical gynaecologic examination. The study was approved by the ethics policy committee of the hospital and was conducted in accordance with the revised 1979 Helsinki Declaration.

\section{Specimen collection}

After cleaning the endocervix with a sterile cotton swab in order to remove mucosal secretions, endocervical specimens were obtained. A sterile speculum was inserted into the vagina to facilitate the exposure of the cervical os. Specimens were collected by rotation in order to collect a sufficient amount of cells and thus increase the frequency of recovery. Care was taken to prevent the contact of the swab with the vaginal epithelium.

\section{Chlamydial antigen detection}

The Chlamydia trachomatis antigen was detected by enzymelinked immunosorbent assay (Biorad, USA). A Chlamydia swab collection kit (Biorad, USA) was used for sample collection. It contained one small Dacron swab on a stainless steel/plastic shaft for collecting specimens, and one tube containing phosphate buffered saline (PBS) for transporting the specimen to the laboratory. After collecting the specimens, they were stored at $20{ }^{\circ} \mathrm{C}$ until use. Chlamydia microplate EIA is a monoclonal antibody based on qualitative enzyme immunoassay for direct detection of chlamydial organisms in adult urogenital specimens. The samples were processed weekly.

\section{Cytological analysis}

Cervical smears were collected using a cytobrush ( CervexBrush $^{\circledR}$ Greece); in patients with cervical stenosis, a cytobrush ${ }^{\circledR}$ (Medscand, Geneva, Switzerland) was used. The staining of thinlayer preparations was performed with the Papanicolaou method, and the inflammatory character of the preparation was examined. All Pap smear results were coded using Bethesda terminology, screened routinely, and independently reviewed to confirm eligibility to this study. Pap smears reported as negative or as with minor reactive changes were grouped together as normal. Pap smears reported as LSIL or HSIL were grouped together as abnormal. If a Pap smear was reported as squamous cells of undetermined significance (ASCUS), the Pap-smear was repeated until the diagnosis was achieved in accordance with recommended practice.

\section{Microbiological assessment}

Specimens for bacterial culture were incubated in blood \& chocolate supplemented Difco ${ }^{\circledR}$ agar plates for $72 \mathrm{~h}$, using selective and non-selective culture media for C. albicans, C. vaginalis and anaerobic bacteria. Specimens were examined by direct phase-contrast microscopy (for detection of Trichomonas or $C$. albicans). Specimens for mycological assessment were cultured in Sabouraud agar plates; specimens for mycoplasma assessment were inoculated in (DNA-PPLO) selective culture media, and a urea test was performed for detecting Mycoplasma hominis.

\section{Colposcopic evaluation}

Colposcopy (Zeiss Lens-Germany) was performed in all patients after the application of $3-5 \%$ acetic acid. The location and appearance of the transformation zone was documented on a standard colposcopy form. Colposcopic abnormalities such as acetowhite alterations, punctuation, mosaic vascular pattern, and lugol uptake (Schiller test) were noted. The cervix was examined with a green filter (16X) to assess wheher atypical vessels were present; punch biopsies were collected from macroscopically visible lesions (posterior lesions were biopsied first). Colposcopy was evaluated as satisfactory when the transformation zone was visualized, while endocervical curettage was performed when the Pap smear had shown atypia or when the transformation zone was not visualized (Colposcopy was considered as non-satisfactory). In cases of HGSIL (CIN II-III), cervical conization and abdominal hysterectomy were performed according to FIGO guidelines and personal request of the patient in respect of preservation of fertility.

\section{Statistical analysis}

The data were entered in Excel and analyzed using the Statistical Package for Social Sciences (SPSS Inc, Chicago, IL, USA). Initially, a descriptive analysis was performed as to the main sociodemographic characteristics, clinical characteristics, sexual behaviour of the participants, and their related risk factors. The patients were divided into two groups, namely group I with positive reactions for Chlamydia trachomatis in cervical smear or urethra, and group II with negative results. The statistical significance of differences in proportions was performed using Fisher's exact test or $\chi^{2}$ test. A $p$ value $<0.05$ was considered statistically significant.

\section{Results}

The demographic characteristics and sexual history of the study population are outlined in Table 1. Amongst the 110 women symptomatic for cervicitis, 41 patients $(37.2 \%)$ were found to be positive for Chlamydia trachomatis antigen, and were thus categorised in Group I (positive for chlamydial infection). Sixty-nine patients $(62.8 \%)$ were negative for Chlamydia trachomatis antigen, and thus placed in Group II (negative for chlamydial infection). The age at first sexual intercourse for group I was $18.2+2.42$, whilst for group II it was $19.1+2.94(\mathrm{p}<0.10)$, a small statistical significance. The number of sexual partners in the group with positive detection of Chlamydia trachomatis antigen stood at $4.1 \pm 3.12$, while in group II it was $2.5 \pm 2.57(\mathrm{p}<0.01)$. In total, the women's' clinical characteristics at admission are outlined in Table 2. Increased vaginal discharge was revealed in $85.5 \%$ of patients in group I,

Tab. 1. Demographic characteristics and sexual history, p-value $<0.05$ : statistically significant.

\begin{tabular}{lccc}
\hline & $\begin{array}{c}\text { Group I } \\
\text { (infected n=41) }\end{array}$ & $\begin{array}{c}\text { Group II } \\
\text { (not infected n=69) }\end{array}$ & $\begin{array}{c}\text { P value } \\
\mathrm{p}<0.05\end{array}$ \\
\hline Age & $33.1 \pm 6.76$ & $33.3 \pm 8.24$ & 0.10 \\
$\begin{array}{l}\text { Age at first sexual } \\
\text { intercourse }\end{array}$ & $18.2+2.42$ & $19.1+2.94$ & $<0.10$ \\
$\begin{array}{l}\text { Number of sexual } \\
\text { partners }\end{array}$ & $4.1 \pm 3.12$ & $2.5 \pm 2.57$ & $<0.01$ \\
$\begin{array}{l}\text { Frequency of sexual } \\
\text { intercourses }\end{array}$ & $1.45 \pm 1.70$ & $2.03 \pm 1.44$ & 0.10 \\
\hline
\end{tabular}


Tab. 2. Clinical characteristics of the examined groups of patients, p-value $<0.05$ : statistically significant.

\begin{tabular}{lccc}
\hline & $\begin{array}{c}\text { Group I } \\
\text { (infected n=41) }\end{array}$ & $\begin{array}{c}\text { Group II } \\
\text { (not infected n=69) }\end{array}$ & $\begin{array}{c}\text { P value } \\
\mathrm{p}<0.05\end{array}$ \\
\hline Vaginal Discharge & $85.5 \%$ & $62.3 \%$ & $<0.05$ \\
Frequent pelvic pain & $60.0 \%$ & $47.8 \%$ & 0.10 \\
Vaginal bleeding during & $30.0 \%$ & $15.9 \%$ & 0.10 \\
sexual intercourse & & $38.2 \%$ & $<0.05$ \\
Vaginal pain during & $60.0 \%$ & & \\
sexual intercourse & & $39.1 \%$ & 0.10 \\
Dysmenorrhoea & $37.5 \%$ & $24.6 \%$ & $<0.05$ \\
Dysuric complaints & $45.0 \%$ & &
\end{tabular}

Tab. 3. Colposcopic findings in the examined Group I \& Group II; $P$ value $<0.05$ statistically significant.

\begin{tabular}{|c|c|c|c|}
\hline & $\begin{array}{c}\text { Group I } \\
\text { (infected } n=41 \text { ) }\end{array}$ & $\begin{array}{c}\text { Group II } \\
\text { (not infected } n=69 \text { ) }\end{array}$ & $\begin{array}{l}\mathrm{P} \text { value } \\
\mathrm{p}<0.05\end{array}$ \\
\hline Cervicitis & $94.9 \%$ & $71.0 \%$ & $<0.01$ \\
\hline $\begin{array}{l}\text { Cylindric epithelium with } \\
\text { inflammation (Ectropion) }\end{array}$ & $97.5 \%$ & $75.1 \%$ & $<0.01$ \\
\hline \multicolumn{4}{|l|}{$\begin{array}{l}\text { Squamous metaplasia } \\
\text { (Trans. zone) }\end{array}$} \\
\hline Immature & $30.0 \%$ & $52.9 \%$ & \\
\hline Mature & $17.5 \%$ & $19.1 \%$ & \\
\hline Atypical & $52.5 \%$ & $27.9 \%$ & $<0.05$ \\
\hline Leukoplakia & $2.9 \%$ & $1.7 \%$ & 0.10 \\
\hline Mosaic alteration & $41.0 \%$ & $18.5 \%$ & $<0.05$ \\
\hline Punctuation & $31.6 \%$ & $21.9 \%$ & 0.10 \\
\hline Inflammation & $95.0 \%$ & $69.6 \%$ & $<0.01$ \\
\hline \multicolumn{4}{|l|}{ Acetowhite alterations } \\
\hline Weak & $27.5 \%$ & $48.5 \%$ & \\
\hline Mild & $45.0 \%$ & $32.4 \%$ & \\
\hline Intense & $27.5 \%$ & $19.1 \%$ & 0.10 \\
\hline Decreased lugol uptake & $43.8 \%$ & $30.2 \%$ & 0.10 \\
\hline Length of lesion (in $\mathrm{cm}$ ) & $1.77 \pm 1.361$ & $1.55 \pm 1.17$ & 0.10 \\
\hline \multicolumn{4}{|l|}{ Vessels formation } \\
\hline Normal & $24.3 \%$ & $54.5 \%$ & \\
\hline Neovascularisation & $70.3 \%$ & $39.4 \%$ & $<0.01$ \\
\hline Atypical & $5.4 \%$ & $6.1 \%$ & \\
\hline
\end{tabular}

Tab. 4. Cytological results in Chlamydia Trachomatis populations.

\begin{tabular}{lcc}
\hline & $\begin{array}{c}\text { Group I } \\
\text { (infected } \mathrm{n}=41)\end{array}$ & $\begin{array}{c}\text { Group II } \\
\text { (not infected } \mathrm{n}=69)\end{array}$ \\
\hline Bethesda diagnosis $(\mathrm{n}=110)$ & & \\
inflammatory specimens & $6(5.4 \%)$ & $2(1.8 \%)$ \\
Normal $\mathrm{n}=86$ & $29(26.3 \%)$ & $57(51.8 \%)$ \\
LGSIL & $7(6.3 \%)$ & $4(3.6 \%)$ \\
CIN-II & $2(1.8 \%)$ & $2(1.8 \%)$ \\
CIN-III & $4(3.6 \%)$ & $4(3.6 \%)$ \\
Invasive Ca & 0 & $1(0.9 \%)$ \\
\hline
\end{tabular}

while in group II, the percentage was $62.3 \%(\mathrm{p}<0.05)$. Women in the chlamydia-infected group presented a larger percentage of dyspareunia (60\%), and in contrast with group II, the dysuric complaints in group I were more common $(24.6 \%$; $<<0.05)$.

The colposcopic findings in the examined groups are presented in Table 3. Ectropion incidence was higher in group I than in group II with a statistically significant result. The chlamydia-positive group revealed a higher incidence of atypical squamous metaplasia
Tab. 5. Results of culture samples for STDs.

\begin{tabular}{lccc}
\hline & Group I & Group II & P value \\
\hline Aerobic & $10.5 \%$ & $10.1 \%$ & 0.10 \\
Anaerobic & $30.8 \%$ & $20.3 \%$ & 0.10 \\
Trichomonas & 23.7 & $11.6 \%$ & 0.10 \\
Fungi & $20.0 \%$ & $27.5 \%$ & 0.10 \\
Mycoplasma & $43.6 \%$ & $29.0 \%$ & 0.10 \\
\hline
\end{tabular}

(52.5\% vs $27.9 \%)$. Another clinical finding with strong statistical significance was the observation of cervical neovascularisation in group I ( $70.3 \%$ vs $39.4 \% ; \mathrm{p}<0.01)$, and mosaic alteration $(40 \%$ vs $18 \% ; \mathrm{p}<0.05)$. Cytological assessment of the obtained cervical smears is presented in Table 4. In total, 86 (78 \%) cervical smears were found to be normal, and 24 ( $22 \%)$ were abnormal. Amongst the women with positive ELISA for chlamydia, a higher proportion of LGSIL (6.3\% vs $3.6 \%$ ) was observed (Tab. 5).

\section{Discussion}

Chlamydial infection is the most common curable bacterial sexually transmitted infection (11). Opportunistic screenings of specific target populations attending many health settings have been suggested in a bid to reduce the prevalence of the disease (4). Sexually active young women are at highest risk of chlamydial infection while women aged 24 years or younger are more than five times as likely as women older than 30 to be infected (12). Other risk factors include a history of chlamydial infection or other sexually transmitted infections, namely new or multiple sexual partners and inconsistent condom use. Risk factors for pregnant women are the same as those for non-pregnant women (13). In consideration of this, a test with high sensitivity would have an important role to play in detecting CT. It was speculated that CT was an independent risk factor for progression from LGSIL to HGSIL and eventual cervical cancer (14). CT infection has been shown to be more frequent in women with CIN than in women with normal cytology $(5,15)$. Other studies however do not support a relationship between CT and CIN or abnormal cervical cytology (16, 17). Chlamydial cervicitis has been suspected to be a risk factor for SIL (Squamous intraepithelial neoplasia) and cervical cancer on the basis of case control comparisons of serological tests but after adjusting for HPV (Human Papilloma virus) infection, the risk of cervical cancer associated with chlamydial seropositivity was considered low $(\mathrm{OR}=2)(18)$.

Blackwell et al (19) found that the prevalence rate for age was $68 \%$ in women younger than 30 years. In those screened over 30 years, the prevalence of CT infection was $0.66 \%$. It should be noted that $58 \%$ of those with positive results were without symptoms. This demonstrates that the screening of symptomatic women only would miss the majority of patients with positive results. In our study there was no statistical significance between the two groups in regard of age and frequency of sexual intercourses, and we also found that the age at first sexual intercourse (18.2 \pm 2.42$)$ and the number of sexual partners $(4.1 \pm 3.12)$ are risk factors for $\mathrm{CT}$ infections. The main clinical characteristics of women suffering from chlamydial infection were vaginal discharge, pain during 


\section{$357-360$}

sexual intercourse, and dysuric complaints. Our findings highlight the routine $\mathrm{CT}$ screening particularly for early detection of genital infection in order to reduce the morbidity and resulting complications. In most cases, the first indication of existence of infection due to Chlamydia trachomatis in a patient is provided by her clinical symptomatology. For this reason, it is necessary to obtain a complete history and a detailed clinical examination of the patient. The findings of Papanicolaou smears for these women support the evidence of CT infections but they are not absolutely diagnostic. They can easily be performed and incorporated into everyday use but they require an experienced physician to interpret the results. As to the usefulness of histologic examination for the diagnosis of chlamydial infections, we concluded that this is limited. As observed in five of our cases, the right treatment can beneficially affect chlamydial infection of the cervix, as well as the progress of cellular atypia. Chlamydial infections meet the general prerequisite for disease prevention by screening since they are highly prevalent, associated with significant morbidity, can be diagnosed, and are treatable (2). We believe that CT infection is a severe infection of female genitalia with all mentioned related complications and should be faced with increased responsibility by the physician, who should employ all the above mentioned diagnostic methods at his disposal. In our opinion, it is ELISA that should be especially used. Unfortunately, the methods mentioned are not sufficient to detect CT and thus for highly reliable conclusions additional and more sensitive methods are needed (polymerase chain reaction or nucleic acid amplification test).

\section{References}

1. Department of Health. Chlamydia Trachomatis. Summary and Conclusions of CMO's expert Advisory Group. London, UK: Department of Health, 1998

2. Paavonen J, Eggert-Kruse W. Chlamydia Trachomatis:impact on human reproduction. Human Reproduction Update1999; (5): 433-447.

3. Lindsay DT, Trounson AO, Anderson AN. Lancet 1994; 334: 15391544.

4. Schachter, J., Hill, E.C., King, E.B. et al. Chlamydia trachomatis and cervical neoplasia. J Am Med Ass 1982; 248: 2134-2138.

5. De Sanjose S, Munoz N, Bosch FX, Reimann K, Pedersen NS, Orfila

$\mathbf{J}$ et al. Sexually transmitted agents and cervical neoplasia in Columbia and Spain. Intern J Cancer 1994; 56: 358-363.
6. Takac I, Gorisek B. Chlamydia trachomatis infection in women with and without cervical intraepithelial neoplasia. Int J STD AIDS 1999; 10 : 331-333.

7. Center for Disease Control Prevention (CDC) Sexually Transmitted Disease Surveilance Report. 2000, Atlanta USA.

8. Stamm WE. Harrison's Principles of Internal Medicine. 15th ed. Columbus, OH: McGraw-Hill, 2001.

9. Morre SA, Rozendaal L, van Valkengoed IGM et al. Urogenital Chlamydia trachomatis serovars in men and women with a symptomatic or asymptomatic infection: An association with clinical manifestations? J Clin Microbiol 2000; 38: 2292-2296.

10. Kurman RJ, Henson DE, Herbst AL et al. Interim guidelines for management of abnormal cervical cytology. The 1992 National Cancer Institute Workshop. J Am Med Ass 1994; 271: 1866-1869.

11. Gaydos CA, Howell MR, Pare B et al. Chlamydia trachomatis infections in Female military recruits. N Engl J Med 1998; 339: 739-744.

12. Svensson LO, Mares I, Olsson SE, Nordstrom ML. Screening for Chlamydia trachomatis infection in women and aspects of the laboratory diagnostics. Acta Obstet Gynecol Scand 1991; 70: 587-590.

13. Meyers DS, Halvorson H, Luckhaupt S. Screening for chlamydial infection: an evidence update for the U.S. Preventive Services Task Force. Ann Intern Med 2007; 147: 135-142.

14. Pimenta JM, Catchpole M, Rogers PA, Hopwood J, Randall S, Mallinson $\mathrm{H}$ et al. Opportunistic screening for genital chlamydial infection.II. Prevalence among healthcare attenders, outcome and evaluation of positive cases. Sexually Transmitted Infect 2003; 79: 22-27.

15. Dunlop E, Carner A, Darouga r S, Treharne J. Colposcopy, biopsy and cytology results in women with Chlamydial cervicitis. Genitourin Med 1989; 65: 22-31.

16. Boyle DC, Smith JR. Infection and cervical intraepithelial neoplasia. Int J Gynecol Cancer 1999; 9 (3): 177-186.

17. Paavonen J, Lehtinen M. Interactions between human papillomavirus and other sexually transmitted agents in the etiology of cervical cancer. Curr Opin Infect Dis 1999; 12: 67-71.

18. Hsieh CY, You SL, Kao CL, Chen CJ. Reproductive and infectious risk factors for invasive cervical cancer in Taiwan. Anticancer Res 1999; 19 (5C): 4495-4500.

19. Blackwell A, Linton D, Emery S, Calvert J. Chlamydia trachomatis infection in a colposcopy unit: an audit of a fast trackreferral system for infected patients to a genitourinary medicine department and a survey of patients' demography, clinical findings and partner details. Int J STD AIDS 2003; 14 (10): 661-664. 